\title{
SU(3) Symmetry in hafnium isotopes with even neutron $\mathrm{N}=\mathbf{1 0 0 - 1 0 8}$
}

\author{
I. Hossain ${ }^{1}$, Fadhil I. Sharrad ${ }^{2}$, Huda H. Kassim ${ }^{3}$, Amir A. Mohammed-Ali ${ }^{4}$, A. S. Ahmed \\ ${ }_{1,5}$ Department of Physics, Rabigh College of Science \& Arts, King Abdulaziz University, Saudi Arabia \\ ${ }^{2,3,4}$ Department of Physics, College of Science, Kerbala University, Iraq \\ ${ }^{2}$ College of Health and Medical Technology, Al-Ayen University, Iraq
}

\begin{tabular}{l}
\hline Article Info \\
\hline Article history: \\
Received Dec 19, 2019 \\
Revised Feb 25, 2020 \\
Accepted Apr 2, 2020 \\
\hline
\end{tabular}

Keywords:

$\mathrm{HF}$

Energy level

Reduced transition probabilities

Deformation parameter

\begin{abstract}
In this paper, we have reviewed the calculation of ground states energy level up to spin $14+$, electric quadrupole moments up to spin $12+$, and reduced transition probabilities of Hafnium isotopes with even neutron $\mathrm{N}=100-108$ by Interacting Boson Model (IBM-1). The calculated results are compared with previous available experimental data and found good agreement for all nuclei. Moreover, we have studied potential energy surface of those nuclei. The systematic studies of quadrupole moments, reduced transition strength, yrast level and potential energy surface of those nuclei show an important property that they are deformed and have dynamical symmetry SU(3) characters.
\end{abstract}

This is an open access article under the CC BY-SA license.

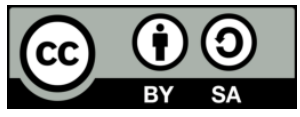

\section{Corresponding Author:}

I. Hossain,

Department of Physics, Rabigh College of Science \& Arts,

King Abdulaziz University,

21911 Rabigh, Saudi Arabia.

Email: mihossain@kau.edu.sa

\section{INTRODUCTION}

The interacting boson model offers a simple Hamiltonian, capable of describing collective nuclear properties across a broad range of nuclei, based on general algebraic group theoretical techniques which have also recently found application in problems in atomic, molecular, and high energy physics [1-4]. The three limiting symmetries of this Hamiltonian, $\mathrm{U}(5), \mathrm{SU}(3)$, and $\mathrm{O}(6)$, correspond to the geometrical shapes, spherical vibrator, symmetric rotor, and $\gamma$-unstable rotor, respectively [5]. The even-mass Hafnium ${ }^{172-180} \mathrm{Hf}$ isotope shave been extensively investigated experimentally using a wide variety of reactions. The excited states in the even-even ${ }^{172-180} \mathrm{Hf}$ isotopes have been investigated from $\left(\gamma, \gamma^{\prime}\right),(\mathrm{d}, \mathrm{p}),(\alpha, 2 \mathrm{n} \gamma),(\alpha, 4 \mathrm{n} \gamma),(\mathrm{n}, \gamma)$, $\left(\mathrm{n}, \mathrm{n}^{\prime} \gamma\right)$ and Coulomb excitation reactions which gave information about the experimental energy levels and the electromagnetic transition probabilities B(E2) in these isotopes [6-15].

In this study, the calculations of energy levels of even-even ${ }^{172-180} \mathrm{Hf}$ isotopes have been done by using interacting boson model. The ground state band, the reduce probabilities of $E 2$ transitions $\left(B(E 2)\right.$ values), and electric quadrupole moment $\mathrm{Q}_{\mathrm{L}}$ are calculated and compared with available experimental data. 


\section{THEORETICAL MODEL}

\subsection{Interacting boson model (IBM)}

The IBM-1 Hamiltonian can be expressed as [16, 17]:

$$
\begin{aligned}
& \mathrm{H}=\varepsilon_{S}\left(s^{\dagger} . \tilde{s}\right)+\varepsilon_{d}\left(d^{\dagger} . \tilde{d}\right)+\sum_{L=0,2,4} \frac{1}{2}(2 L+1)^{\frac{1}{2}} C_{L}\left[\left[d^{\dagger} \times d^{\dagger}\right]^{(L)} \times[\tilde{d} \times \tilde{d}]^{(L)}\right]^{(0)}+\frac{1}{\sqrt{2}} v_{2}\left[\left[d^{\dagger} \times\right.\right. \\
& \left.\left.d^{\dagger}\right]^{(2)} \times[\tilde{d} \times \tilde{s}]^{(2)}+\left[d^{\dagger} \times s^{\dagger}\right]^{(2)} \times[\tilde{d} \times \tilde{d}]^{(2)}\right]^{(0)}+\frac{1}{2} v_{0}\left[\left[d^{\dagger} \times d^{\dagger}\right]^{(0)} \times[\tilde{s} \times \tilde{s}]^{(0)}+\left[s^{\dagger} \times s^{\dagger}\right]^{(0)} \times\right. \\
& \left.[\tilde{d} \times \tilde{d}]^{(0)}\right]^{(0)}+\frac{1}{2} u_{0}\left[\left[s^{\dagger} \times s^{\dagger}\right]^{(0)} \times[\tilde{s} \times \tilde{s}]^{(0)}\right]^{(0)}+u_{2}\left[\left[d^{\dagger} \times s^{\dagger}\right]^{(2)} \times[\tilde{d} \times \tilde{s}]^{(2)}\right]^{(0)}
\end{aligned}
$$

Then the IBM-1 Hamiltonian in (1) can be written in general form as [18-20]:

$$
\widehat{H}=\varepsilon \hat{n}_{d}+a_{0} \hat{P} \cdot \hat{P}+a_{1} \hat{L} \cdot \hat{L}+a_{2} \hat{Q} \cdot \hat{Q}+a_{3} \widehat{T}_{3} \cdot \widehat{T}_{3}+a_{4} \widehat{T}_{4} \cdot \widehat{T}_{4}
$$

Where $\hat{n}_{d}=\left(\mathrm{s}^{\dagger}, \mathrm{d}^{\dagger}\right)$ isthe total number of $\mathrm{d}_{\text {boson }}$ operator, $\hat{p}=1 / 2[(\tilde{d} . \tilde{d})-(\tilde{s} . \tilde{s})]$ is the pairing operator, $\hat{L}=\sqrt{10}\left[d^{\dagger} \times \tilde{d}\right]^{1}$ is the angular momentum operator, $\hat{Q}=\left[d^{\dagger} \times \tilde{s}+s^{\dagger} \times \tilde{d}\right]^{(2)}+\chi\left[d^{\dagger} \times \tilde{d}\right]^{(2)}$ is the quadrupole operator $\left(\chi\right.$ is the quadrupole structure parameter and take the values 0 and $\left.\pm \frac{\sqrt{7}}{2}[19,20]\right), \widehat{T}_{r}=\left[d^{\dagger} \times \tilde{d}\right]^{(r)}$ is the octoupole ( $\mathrm{r}=3$ ) and hexadecapole ( $\mathrm{r}=4)$ operator, and $\mathrm{e}=\mathrm{e}_{d}-\mathrm{e}_{s}$ is the boson energy. The parameters are $a_{0}$ the strength of the pairing, $a_{1}$ angular momentum, $a_{2}$ quadrupole, $a_{3}$ octoupole and $a_{4}$ hexadecapole interaction between the bosons.

\section{RESULTS AND DISCUSSION}

\subsection{Ground state band}

In Figure 1 shows that the even-even ${ }^{174-180} \mathrm{Hf}$ isotopes have a rotational (deformed nuclei) dynamical symmetry SU(3). The rotational limit of the IBM-1 has been applied for the even-even ${ }^{172-180} \mathrm{Hf}$ isotopes due to the values of the $E_{4_{1}^{+}} / E_{2_{1}^{+}} \operatorname{ratio}\left(E_{4_{1}^{+}} / E_{2_{1}^{+}}=3.33\right)[19,20]$. The best fitting for the Hamiltonian parameters are presented in Table 1 which gives the best agreement with the experimental data [21-26]. In the framework of the IBM-1, the isotopic chains of Hafnium $(Z=72)$ nuclei, having a number of proton-bosons holes 5 , a number of neutron-bosons holes are $(9,10,11)$ for even ${ }^{172-176} \mathrm{Hf}$, and $(10,9)$ for even ${ }^{178-180} \mathrm{Hf}$ isotopes, respectively.

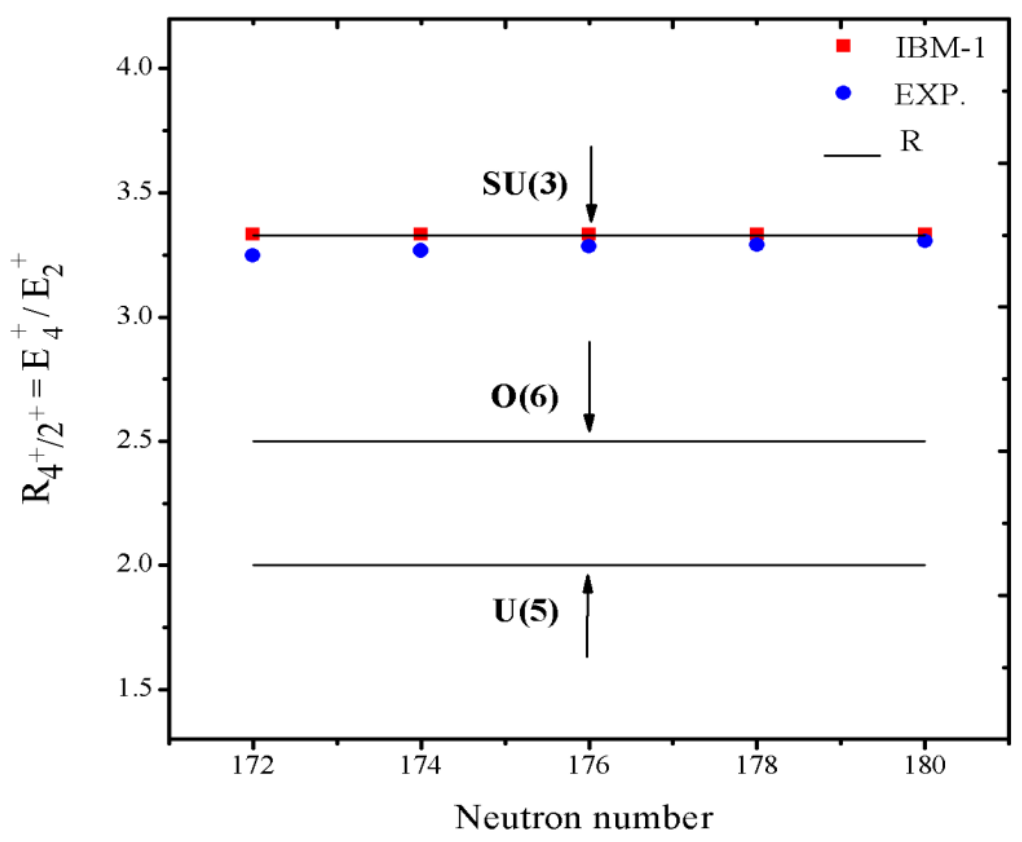

Figure 1. Comparison the IBM-1 calculations with the available experimental data [22-28] of the $E_{4_{1}^{+}} / E_{2_{1}^{+}}$ ratio for even-even ${ }^{172-180} \mathrm{Hf}$ nuclei. 
Table 1.The parameters of even-even ${ }^{172-180} \mathrm{Hf}$ nuclei.

\begin{tabular}{ccccccccc}
\hline Nuclei & $N$ & $\begin{array}{c}\mathrm{e} \\
\mathrm{MeV}\end{array}$ & $\begin{array}{c}a_{0} \\
\mathrm{MeV}\end{array}$ & $\begin{array}{c}a_{1} \\
\mathrm{MeV}\end{array}$ & $\begin{array}{c}a_{2} \\
\mathrm{MeV}\end{array}$ & $\begin{array}{c}a_{3} \\
\mathrm{MeV}\end{array}$ & $\begin{array}{c}a_{4} \\
\mathrm{MeV}\end{array}$ & \multicolumn{1}{c}{$\mathrm{CHQ}(\chi)$} \\
\hline${ }^{172} \mathrm{Hf}$ & 14 & 0.00 & 0.00 & 0.024 & -0.021 & 0.00 & 0.00 & -1.33 \\
${ }^{174} \mathrm{Hf}$ & 15 & 0.00 & 0.000 & 0.023 & -0.019 & 0.00 & 0.00 & -1.33 \\
${ }^{176} \mathrm{Hf}$ & 16 & 0.00 & 0.000 & 0.020 & -0.0242 & 0.00 & 0.00 & -1.33 \\
${ }^{178} \mathrm{Hf}$ & 15 & 0.00 & 0.000 & 0.021 & -0.0258 & 0.00 & 0.00 & -1.33 \\
${ }^{180} \mathrm{Hf}$ & 14 & 0.00 & 0.000 & 0.021 & -0.0268 & 0.00 & 0.00 & -1.33 \\
\hline
\end{tabular}

Figure 2 indicates the energy of ground state band in experimental and theoretical data. This figure has shown the IBM-1 calculations for ground band (energies, spin and parity) in good agreement with those of the experimental data [22-28].
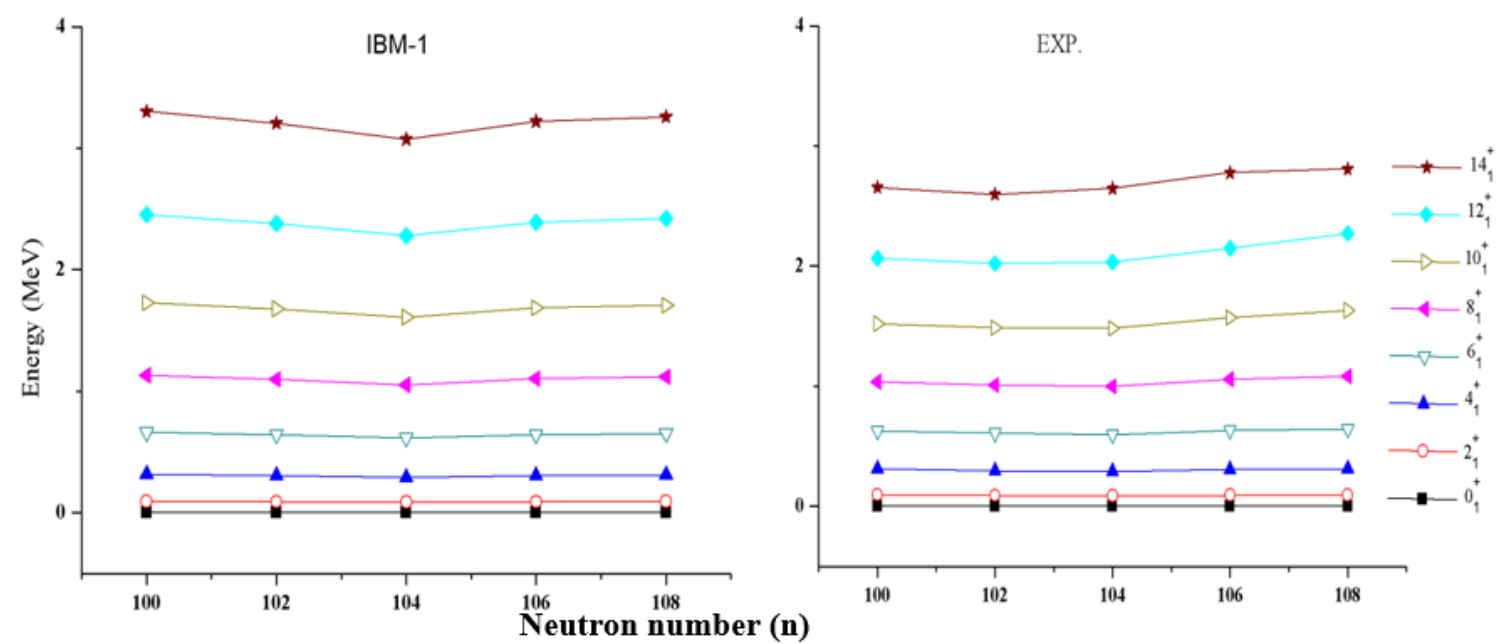

Figure 2. Ground state energy level in the IBM-1 calculations and experimental data [22-28] of even-even ${ }^{172-180} \mathrm{Hf}$ isotopes.

\section{2. $B(E 2)$ and $Q_{L}$ value}

The general form of the electromagnetic transitions operator in IBM-1is [19, 20, 29]:

$$
\widehat{T}^{(\mathrm{L})}=\gamma_{0}\left[\hat{s}^{+} \times \hat{s}^{\sim}\right]^{(0)}+\alpha_{2}\left[\hat{d}^{+} \times \hat{s}^{\sim}+\hat{s}^{+} \times \hat{d}^{\sim}\right]^{(2)}+\beta_{L}\left[\hat{d}^{+} \times \hat{d}^{\sim}\right]^{(L)}
$$

Where $\gamma_{0}, \alpha_{2}$ and $\beta_{\mathrm{L}}(\mathrm{L}=0,1,2,3,4)$ are parameters specifying the various terms in the corresponding operators. Equation (4) yields transition operators for E2 transitions with appropriate values of the corresponding parameters.[19]:

$$
T^{E 2}=\alpha_{2}\left[d^{\dagger} s+s^{\dagger} d\right]^{(2)}+\beta_{2}\left[d^{\dagger} d\right]^{(2)}
$$

Where $\left(s^{\dagger}, d^{\dagger}\right)$ and $(s, d)$ are creation and annihilation operators for $\mathrm{s}$ and d bosons, respectively [30], while $\alpha_{2}$ and $\beta_{2}$ are two adjustable parameters that measure the strength of each term. The electric transition probabilities, B(E2) values are defined in terms of reduced matrix elements by Iachello and Arima (1987) as [20,29]:

$$
B\left(E 2, J_{i} \rightarrow J_{f}\right)=\frac{1}{2 J_{i}+1}\left|\left\langle J_{f}|| T^{E 2} \| J_{i}\right\rangle\right|^{2}
$$

For the calculations of the absolute $B(\mathrm{E} 2)$ values, the parameters, $\alpha_{2}$ and $\beta_{2}$ of (4), were adjusted according to reproduce the experimental $\mathrm{B}\left(\mathrm{E} 2 ; 2_{1}^{+} \rightarrow 0_{1}^{+}\right)$. Table 2 shows the values of the $\alpha_{2}$ and $\beta_{2}$ parameters, which were obtained in the present calculations. The comparison of calculations values of $\mathrm{B}(\mathrm{E} 2)$ transitions with experimental data[22-29], are given in Table 3, for all isotopes under study. 
Table 2. Effective charge (in eb) used to reproduce B(E2)valuesfor ${ }^{172-180} \mathrm{Hf}$ nuclei.

\begin{tabular}{cccc}
\hline $\mathrm{A}$ & $\mathrm{N}$ & $\alpha_{2}$ & $\beta_{2}$ \\
\hline${ }^{172} \mathrm{Hf}$ & 14 & 0.1004 & -0.2969 \\
${ }^{174} \mathrm{Hf}$ & 15 & 0.0940 & -0.2780 \\
${ }^{176} \mathrm{Hf}$ & 16 & 0.0980 & -0.2898 \\
${ }^{178} \mathrm{Hf}$ & 15 & 0.0980 & -0.2898 \\
${ }^{180} \mathrm{Hf}$ & 14 & 0.1038 & -0.3071 \\
\hline
\end{tabular}

Table 3.The $\mathrm{B}(\mathrm{E} 2)$ values for ${ }^{172-180} \mathrm{Hf}$ nuclei (in $\mathrm{e}^{2} \mathrm{~b}^{2}$ ).

\begin{tabular}{|c|c|c|c|c|c|c|c|c|c|c|}
\hline$J_{i} \rightarrow J_{f}$ & IBM-1 & EXP. & $\begin{array}{r}\text { IBM-1 } \\
17\end{array}$ & EXP. & IBM-1 & EXP. & IBM-1 & EXP. & $\begin{array}{r}\text { IBM-1 } \\
18\end{array}$ & EXP. \\
\hline $2_{1}^{+} \rightarrow 0_{1}^{+}$ & 0.8748 & 0.8754 & 0.8735 & 0.8775 & 1.0689 & 1.0726 & 0.9493 & 0.9521 & 0.9353 & 0.9361 \\
\hline $2_{2}^{+} \rightarrow 0_{1}^{+}$ & 0.0000 & -- & 0.0000 & 0.0013 & 0.0000 & 0.0005 & 0.0058 & 0.0043 & 0.0073 & -- \\
\hline $2_{2}^{+} \rightarrow 0_{2}^{+}$ & 0.6845 & -- & 0.0053 & -- & 0.0059 & -- & 1.3424 & -- & 1.3208 & 1.3891 \\
\hline $2_{3}^{+} \rightarrow 0_{2}^{+}$ & 0.0270 & -- & 0.7092 & - & 0.8647 & -- & 0.4562 & - & 0.4389 & - \\
\hline $4_{1}^{+} \rightarrow 2_{1}^{+}$ & 1.2353 & - & 1.2351 & -- & 1.5129 & -- & 1.4511 & 1.3746 & 1.4242 & 1.4556 \\
\hline $4_{1}^{+} \rightarrow 2_{2}^{+}$ & 0.0000 & -- & 0.0000 & 0.0416 & 0.0000 & 0.0140 & 0.8797 & -- & 0.8279 & -- \\
\hline $4_{2}^{+} \rightarrow 2_{2}^{+}$ & 0.0294 & -- & 0.4197 & -- & 0.5198 & -- & 1.4776 & 1.4460 & 1.4447 & 1.5099 \\
\hline $6_{1}^{+} \rightarrow 4_{1}^{+}$ & 1.3320 & -- & 1.3352 & -- & 1.6384 & -- & 1.0258 & -- & 0.8430 & -- \\
\hline $6_{2}^{+} \rightarrow 4_{2}^{+}$ & 0.7624 & - & 0.8094 & -- & 1.0047 & -- & 1.4620 & 1.4817 & 1.4219 & 1.4495 \\
\hline $8_{1}^{+} \rightarrow 6_{1}^{+}$ & 1.3512 & - & 1.3596 & -- & 1.6728 & -- & 1.0652 & -- & 0.9466 & -- \\
\hline $8_{2}^{+} \rightarrow 6_{2}^{+}$ & 0.9183 & -- & 0.9438 & -- & 1.1756 & -- & 1.4178 & 1.7140 & 1.3692 & 1.3408 \\
\hline $10_{1}^{+} \rightarrow 8_{1}^{+}$ & 1.3299 & - & 1.3452 & -- & 1.6611 & -- & 1.0499 & -- & 0.9141 & -- \\
\hline $10_{2}^{+} \rightarrow 8_{2}^{+}$ & 0.0278 & -- & 0.9801 & -- & 1.2267 & -- & 1.3593 & -- & 1.3397 & -- \\
\hline $12_{1}^{+} \rightarrow 10_{1}^{+}$ & 1.2806 & - & 1.3045 & - & 1.6185 & -- & 1.2356 & -- & 1.2179 & -- \\
\hline $12_{2}^{+} \rightarrow 10_{3}^{+}$ & 0.9011 & -- & 0.0021 & -- & 0.0022 & -- & 1.2106 & -- & 1.1935 & -- \\
\hline $2_{1}^{+} \rightarrow 2_{1}^{+}$ & 1.2529 & - & 1.2506 & -- & 1.5299 & -- & 1.2012 & -- & 1.1846 & -- \\
\hline $4_{1}^{+} \rightarrow 4_{1}^{+}$ & 1.1390 & -- & 1.1368 & -- & 1.3904 & -- & 1.1964 & -- & 1.1803 & -- \\
\hline $6_{1}^{+} \rightarrow 6_{1}^{+}$ & 1.1162 & - & 1.1139 & - & 1.3618 & -- & 1.1933 & -- & 1.1779 & - \\
\hline $8_{1}^{+} \rightarrow 8_{1}^{+}$ & 1.1078 & -- & 1.1053 & -- & 1.3504 & -- & 0.9493 & 0.9521 & 0.9353 & 0.9361 \\
\hline $10_{1}^{+} \rightarrow 10_{1}^{+}$ & 1.1038 & -- & 1.1008 & -- & 1.3438 & -- & 0.0058 & 0.0043 & 0.0073 & -- \\
\hline $12_{1}^{+} \rightarrow 12_{1}^{+}$ & 1.1015 & -- & 1.0980 & -- & 1.3386 & -- & 1.3424 & -- & 1.3208 & 1.3891 \\
\hline
\end{tabular}

Table 3 shows that, in general, most of the calculated results in IBM-1 reasonably consistent with the available experimental data, except for few cases that deviate from the experimental data.

The quadrupole moment $\left(\mathrm{Q}_{\mathrm{L}}\right)$ is an important property for nuclei that can determine if the nucleus is spherical $(\mathrm{Q}=0)$, deformed oblate $(\mathrm{Q}<0)$ or prolate $(\mathrm{Q}>0)$ shapes. The electric quadrupole moments of the nuclei can be derived from the transition rate $\mathrm{B}\left(\mathrm{E} 2, \mathrm{~L}_{\mathrm{i}} \rightarrow \mathrm{L}_{\mathrm{f}}\right)$ values according to (6) [31]:

$$
\mathrm{Q}_{\mathrm{L}}=[16 \pi / 5]^{1 / 2}[\mathrm{~L}(2 \mathrm{~L}-1) /(2 \mathrm{~L}+1)(\mathrm{L}+1)(2 \mathrm{~L}+3)]^{1 / 2}\left[\mathrm{~B}\left(\mathrm{E} 2, \mathrm{~L}_{\mathrm{i}} \rightarrow \mathrm{L}_{\mathrm{f}}\right)\right]^{1 / 2}
$$

Where $\mathrm{L}$ is the angular momentum. Table 4 presents the calculation of the electric quadrupole moment $\mathrm{Q}_{\mathrm{L}}$ within the framework of IBM-1 for the even-even Hf nuclei. The presented results for $\mathrm{Q}_{\mathrm{L}}$ are compared with previous experimental results [32].

Table 4. The electric quadrupole moment $\mathrm{Q}_{\mathrm{L}}$ (in eb)

\begin{tabular}{|c|c|c|c|c|c|c|c|c|c|c|}
\hline \multirow[b]{2}{*}{$\mathrm{Q}_{\mathrm{L}}$} & IBM-1 & EXP. & IBM-1 & EXP. & IBM-1 & EXP. & IBM-1 & EXP. & IBM-1 & EXP. \\
\hline & \multicolumn{2}{|c|}{${ }^{172} \mathrm{Hf}$} & \multicolumn{2}{|c|}{${ }^{174} \mathrm{Hf}$} & \multicolumn{2}{|c|}{${ }^{176} \mathrm{Hf}$} & \multicolumn{2}{|c|}{${ }^{178} \mathrm{Hf}$} & \multicolumn{2}{|c|}{${ }^{180} \mathrm{Hf}$} \\
\hline $2_{1}^{+}$ & -1.8971 & -- & -1.8953 & - & -2.0963 & $-2.10(2)$ & -1.9759 & $-2.02(2)$ & -1.9616 & $-2.00(2)$ \\
\hline $2_{2}^{+}$ & 1.7001 & -- & 1.7141 & - & 1.9059 & - & 1.7870 & - & 1.7639 & -- \\
\hline $4_{1}^{+}$ & -2.4144 & -- & -2.4121 & - & -2.6676 & - & -2.5147 & - & -2.4966 & -- \\
\hline $6_{1}^{+}$ & -2.6559 & -- & -2.6531 & - & -2.9335 & - & -2.7659 & - & -2.7463 & - \\
\hline $8_{1}^{+}$ & -2.7956 & -- & -2.7924 & - & -3.0865 & - & -2.9111 & - & -2.8908 & - \\
\hline $10_{1}^{+}$ & -2.8868 & -- & -2.8829 & - & -3.1851 & - & -3.0054 & - & -2.9851 & - \\
\hline $12_{1}^{+}$ & -2.9509 & -- & -2.9461 & - & -3.2530 & - & -3.0713 & - & -3.0514 & - \\
\hline
\end{tabular}

\subsection{Potential energy surface (PES)}

In recent years, the potential energy surface by Skyrme mean field method was mapped onto the PES of the IBM Hamiltonian[33-36].The expectation value of the IBM-1 Hamiltonian with the coherent state $(|N, \beta, \gamma\rangle)$ is used to create the IBM energy surface $[20,37]$. The state $|N, \beta, \gamma\rangle$ is a product of boson creation operators $\left(b_{c}^{\dagger}\right)$ over the boson vacuum $|0\rangle$, i.e. 


$$
|N, \beta, \gamma\rangle=1 / \sqrt{N !}\left(b_{c}^{\dagger}\right)^{N}|0\rangle
$$

With

$$
b_{c}^{\dagger}=\left(1+\beta^{2}\right)^{-1 / 2}\left\{s^{\dagger}+\beta\left[\cos \gamma\left(d_{0}^{\dagger}\right)+\sqrt{1 / 2} \sin \gamma\left(d_{2}^{\dagger}+d_{-2}^{\dagger}\right)\right]\right\}
$$

The energy surface, as a function of $\beta$ and $\gamma$, has been given by [10]

$$
E(N, \beta, \gamma)=\frac{N \varepsilon_{d} \beta^{2}}{\left(1+\beta^{2}\right)}+\frac{N(N+1)}{\left(1+\beta^{2}\right)^{2}}\left(\alpha_{1} \beta^{4}+\alpha_{2} \beta^{3} \cos 3 \gamma+\alpha_{3} \beta^{2}+\alpha_{4}\right)
$$

Where the $\alpha_{\mathrm{i}}$ 's are related to the coefficients $C_{L}, v_{2}, v_{0}, u_{2}$ and $u_{0}$ of (1). $\beta$ Measures the total deformation of nucleus, when $\beta=0$, the shape is spherical, and when $\beta \neq 0$ the shape is distorted. $\gamma$ is the amount of deviation from the focus symmetry and correlates with the nucleus. If $\gamma=0$ the shape is prolate, else if $\gamma=$ 60 the shape becomes oblate.

The calculated potential energy surfaces for the even-even ${ }^{172-180} \mathrm{Hf}$ are presented in Figure 3.From this figure all nuclei are deformed and have rotational-like characters. The prolate deformation is deeper than oblate in all nuclei.

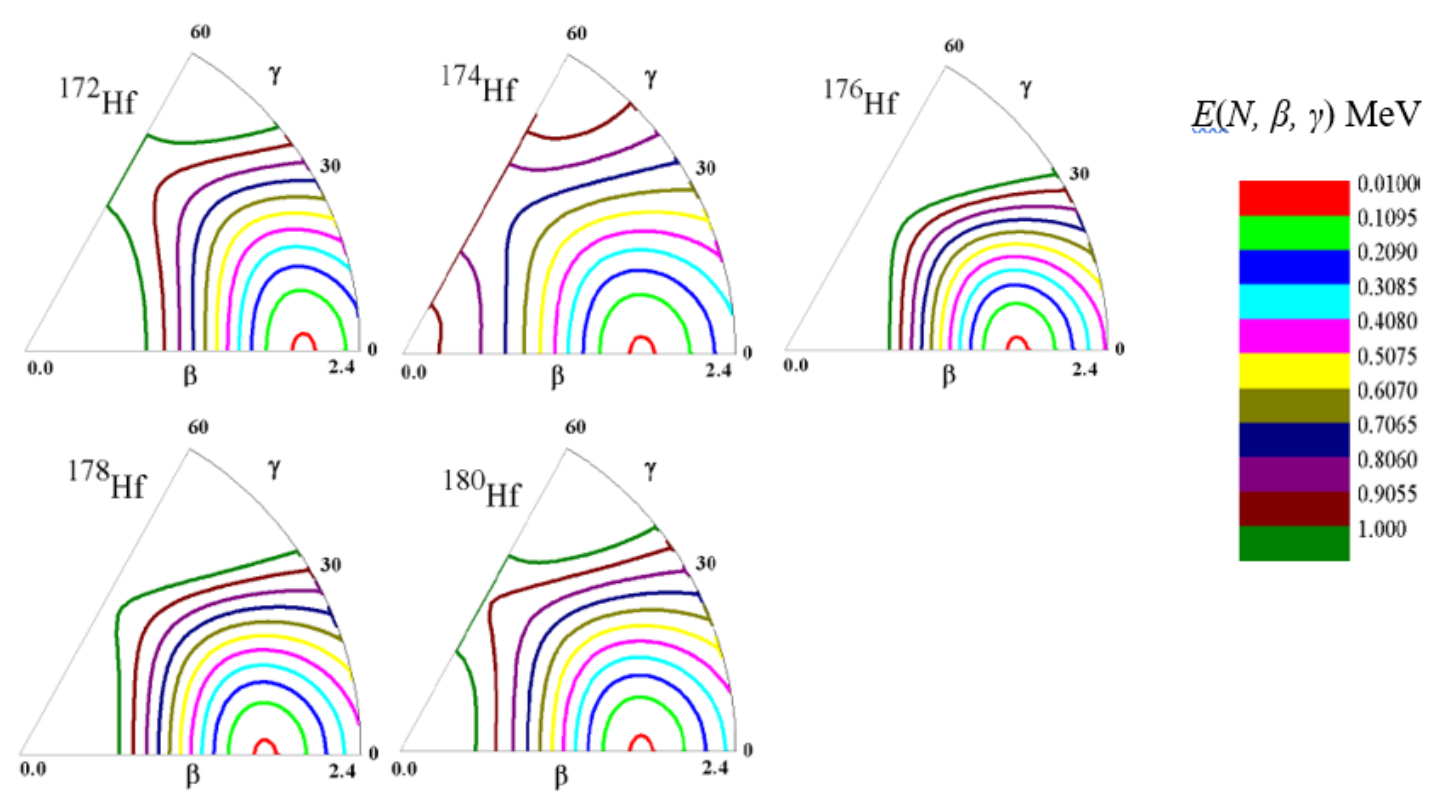

Figure 3. The potential energy surfaces for even-even ${ }^{172-180} \mathrm{Hf}$ nuclei.

\section{CONCLUSION}

We have reviewed theoretical calculations of ${ }^{172-180} \mathrm{Hf}$ isotopes with $\mathrm{N}=100,102,104,106$ and 108 using IBM-1. The even-even ${ }^{176-180} \mathrm{Hf}$ isotopes have bosons total numbers of $14,15,16,15$ and 14 . They were considered fully rotational (fully deformed) nuclei, and the dynamical symmetry of these isotopes is $\mathrm{SU}(3)$. The low-lying ground states, electric transition probabilities $\mathrm{B}(\mathrm{E} 2)$, and electric quadrupole moment $\mathrm{Q}_{\mathrm{L}}$ are obtained for these isotopes using IBM-1 were compared with the available experimentally data. A good agreement was obtained between theoretical IBM-1for all the observable studied. The potential energy surfaces for Hf isotopes shows that all nuclei are deformed and have dynamical symmetry SU(3) characters.

\section{APPENDIX}

PACS numbers: 21.60.Ev, 27.70.+q, 23.20.-g, 23.20. Lv. 


\section{ACKNOWLEDGMENTS}

this work.

We thank University of Kerbala, College of Science, and Department of Physics for supporting

\section{REFERENCES}

[1] Van Roosmalen, O. S., A. E. Dieperinkand, and F. Iachello, "Spectroscopic studies of distorted structure systems in the Vibron model: application to porphyrin and its isotopomers," Chem Phys Lett, vol. 85, pp. 32-36, 1982.

[2] Kellman, Michael E., and David R. Herrick, "Ro-vibrational collective interpretation of super multiplet classifications of intra shell levels of two-electron atoms," Physical Review A, vol. 22, no. 4, pp. 1536, 1980.

[3] Sharrad, et al., "Low-lying states of 184W and 184Os nuclei," Chinese Physics C, vol. 37, no. 3, pp. 034101, 2013.

[4] Otsuka, T., A. Arima, and F. Iachello, "Nuclear shell model and interacting bosons," Nuclear Physics A, vol. 309, no. $1-2$, pp. $1-33,1978$.

[5] Sethi, A., et al., "Inelastic proton scattering from Pt isotopes and the interacting boson model," Physical Review C, vol. 44, no. 2, pp. 700, 1991.

[6] N. Pietralla, et al, "The scissors mode and other magnetic and electric dipole excitations in the transitional nuclei ${ }^{178,180}$ Hf," Nuclear Physics A, vol. 618, no. 1-2, pp. 141-165, 1997

[7] Raymond, K., et al, "K $\mathrm{K}^{\pi}=3^{+}$band at $1862 \mathrm{keV}$ in ${ }^{178} \mathrm{Hf}$," Phys Rev C, vol. 48, no. 2, pp. 911-913, 1993.

[8] J. I. Zaitz and R. K. Sheline, "Single-Neutron-Transfer-Reaction studies of ${ }^{176} \mathrm{Hf}$ and ${ }^{180} \mathrm{Hf}$," Phys. Rev. C, vol. 6 no. 2, pp. 506-516, 1972.

[9] Ejiri, H., and G. B. Hagemann, "Electromagnetic transitions from levels in the beta-vibrational band of 174Hf," Nuclear Physics A, vol. 161, no. 2, pp. 449-470, 1971.

[10] T. L. Khoo, F. M. Bernthal, R. G. H. Robertson, and R. A. Warner, "High-Spin multi quasi-particle yrast traps in ${ }^{176}$ Hf," Phys. Rev. Lett., vol. 37, no. 13, pp. 823-825, 1976.

[11] A.M.I. Hague, et al, "A study of the low-lying states in $178 \mathrm{Hf}$ through the (n, $\gamma$ ) reaction," Nuclear Physics A, vol. 455, no. 2, pp. 231-293, 1986

[12] D. L. Bushnell, D.J. Buss and R.K. Smitter, "180Hf energy levels deduced from thermal and average resonance neutron-capture $\gamma$-ray spectra," Phys. Rev. C, vol. 10, no. 6, pp. 2483-2511, 1974.

[13] I.A. Kondurov, E.M. Korotkikh, Yu.V. Petrovand G.I. Shuljak, "Acceleration of thermal neutrons by isomeric nuclei (180Hfm),” Phys. Lett. B, vol. 106, no. 5, pp. 383-385, 1981.

[14] T. Morikawa, et al, "Coulomb excitation of $174 \mathrm{Hf} \mathrm{K}$-isomer. $\gamma$-ray spectroscopy with high-spin isomer beam," Phys. Lett. B, vol. 350, no. 2, pp. 169-172, 1995.

[15] S. Raman et al., "Transition probability, B(E2) $\uparrow$, from the ground to the first-excited 2+ state of even-even nuclides," Atomic Data and Nuclear Data Tables, vol. 36, no. 1, pp. 1-96, 1987.

[16] Arima, A., \& Iachello, F., "Interacting boson model of collective nuclear states IV. The O (6) limit" Annals of Physics, vol. 123, no. 2, pp. 468-492, 1979.

[17] Küçükbursa, Atalay, et al., "IBM-1 calculations on the even-even 122-128Te isotopes," Mathematical and Computational Applications, vol. 10, no. 1, pp. 9-17, 2005.

[18] A. Arima and F. Iachello, "Interacting boson model of collective nuclear states II. The rotational limit," Annals of Physics, vol. 111, no. 1, pp. 201-238, 1978

[19] R.F. Casten and D.D. Warner, "The interacting boson approximation," Reviews of Modern Physics, vol. 60, no. 2, pp. 389-470, 1988.

[20] F. Iachelloand A. Arima, The Interacting Boson Model, Cambridge: Cambridge University Press, 1987.

[21] I. Hossain, Huda H. Kassim, Mushtaq A. Al-Jubbori, Fadhil I. Sharrad, Said A. Mansour, "Ground States Structure of Ruthenium Isotopes with Neutron $N=60,62$," World Journal of Nuclear Science and Technology, vol. 10, pp. $76-84,2020$.

[22] B. J. Singh, "Nuclear data sheets for A = 172," Nucl. Data Sheets, vol. 75, no. 2, pp. 199-376, 1995.

[23] E. Browne and H. Junde, "Nuclear Data Sheets for A = 174," Nuclear Data Sheets, vol. 87, no. 1, pp. 15-176, 1999.

[24] M.S. Basunia, "Nuclear Data Sheets for A = 176," Nuclear Data Sheets, vol. 107, no. 4, pp. 791-1026, 2006.

[25] E. Achterberg, O.A. Capurro, and G.V. Marti, "Nuclear Data Sheets for A = 178," Nuclear Data Sheets, vol. 110, no. 7, pp. 1473-1688, 2009.

[26] S.C. Wu, and H. Niu, "Nuclear Data Sheets for A = 180," Nuclear Data Sheets, vol. 100, no. 4, pp. 483-705, 2003.

[27] H H Kassim, et al., "Calculation of some of the nuclear properties of even-even 172-176Hf isotopes using IBM-1," J. Natin. Sci. Foundation Sri. Lanka, vol. 46, no. 1, pp. 3-10, 2018.

[28] H H Kassim, et al., "Nuclear structure of Even178-182Hf Isotopes under the Framework of Interacting Boson Model (IBM-1)," Iran J Sci Technol Trans Sci, vol. 42, pp. 993-999, 2018.

[29] Yazar, Harun Resit, and Ümit Erdem., "Nature of excited states of gadolinium isotopes," Chinese Journal of Physics vol. 46, no. 3, pp. 270-277, 2008.

[30] F.X. Xu, C.S. Wu and J.Y. Zeng, "Relations for the coefficients in the $\mathrm{I}(\mathrm{I}+1)$ expansion for rotational spectra," Phys. Rev. C, vol. 40, no. 5, pp. 2337-2341, 1989.

[31] Vidya Devi, HM Mittal, Neha Sharma, "Search of O (6) symmetry in A=120-200 mass region," DAE Symp. Nucl. Phys., vol. 54, pp. 172-173, 2009.

[32] N.J. Stone, "Table of nuclear magnetic dipole and electric quadrupole moments," Atomic Data and Nuclear Data Tables, vol. 90, no. 1, pp. 75-176, 2005. 
[33] L. M. Robledo, et al, "Role of triaxiality in the ground-state shape of neutron-rich Yb, Hf, W, Os and Pt isotopes," J. Phys. G: Nucl. Part. Phys., vol. 36, no. 11, pp. 115104, 2009.

[34] K. Nomura, et al, "Derivation of IBM Hamiltonian for deformed nuclei," J. Phys.: Conf. Ser., vol. 267, pp. 012050, 2011.

[35] I. Bentley and S. Frauendorf, "Microscopic calculation of interacting boson model parameters by potential-energy surface mapping," Phys. Rev. C, vol. 83, no. 6, pp. 064322, 2011.

[36] K. Nomura, et al, "Microscopic formulation of the interacting boson model for rotational nuclei," Phys. Rev. C, "vol. 83, no. 4, pp. 041302, 2011.

[37] F. I. Sharrad, et al., "SHAPE Transition and Collective Excitations in Neutron-Rich 170-178Yb Nuclei," Rom. Journ. Phys., vol. 57, no. 9-10, pp. 1346-1355, 2012. 